\title{
Délibérations du Comité central
}

\section{Lors de ses dernières séances, le Comité central de la FMH a ...}

... pris connaissance du fait qu'en septembre, la FMH a lancé la fabrication de 1200 cartes de professionnel de santé.

... décidé de réduire le nombre de rapports annuels imprimés.

... apporté son soutien à l'Association suisse des neuropsychologues (ASNP) en ce qui concerne «l'admission du diagnostic neuropsychologique ambulatoire dans l'OAMal».

... approuvé les propositions suivantes:

- utilisation du logo FMH et contribution de soutien au «20th IFP World Congress of Psychotherapy» de juillet 2010 organisé par la FMPP en collaboration avec l'International Federation of Psychotherapy;

- parrainage du Swiss eHealth Forum 2010 y compris le droit d'utiliser le logo FMH;

- parrainage de la Swiss medical Student Convention de novembre 2009 à Berne;

- soutien aux projets «Gestion interne des incidents critiques» et «Safety Hotspots et climat de sécurité dans les cabinets de médecine de premier recours en Suisse» de la Fondation pour la sécurité des patients;

- participation financière à une soirée organisée lors de l'assemblée de l'Union Européenne des Médecins Omnipracticiens (UEMO) en juin 2010.

... décidé de participer à une enquête sur la cybersanté (eHealth) des Info Society Days (anciennement: journées télématiques de Berne).

... décidé de signer, avec santésuisse, la conventioncadre sur la création d'une commission de qualité commune.

... accepté de fournir à l'Obsan des données anonymisées pour le projet Monimed dont l'objectif est de développer un modèle de pronostic permettant d'estimer combien de médecins exerceront une activité clinique parmi les nouveaux diplômés.

... pris connaissance du document de base «Transparence de la qualité» du département Données, démographie et qualité (DDQ), ainsi que la prise de position y relative.

... confié au département DDQ le mandat de prendre contact avec l'ASSM au sujet des règles générales de certification des centres de sénologie.

... élu les Drs Hélène Beutler et Adrian Wirthner dans le groupe de pilotage du Groupe de travail Qualité de la FMH.
... approuvé la nomination de Daniel Herren, membre du Comité central, au Conseil de fondation de l'EQUAM.

... accepté le choix de Beat Bumbacher pour succéder à Olivier Kappeler au Comité directeur de medisuisse.

... reçu des informations sur les sujets et projets suivants de l'Institut suisse pour la formation médicale postgraduée et continue (ISFM):

- évaluations: la création d'une plate-forme internet pour les examens de spécialiste permettra de mieux soutenir les sociétés de discipline médicale et d'optimiser ensuite les examens;

- plate-forme de formation continue: ce projet devra, au plus tard au début 2010, être mis sur pied en vue d'améliorer la formation continue et notamment la gestion administrative des sociétés de discipline médicale;

... pris connaissance du fait que santésuisse est désormais disposée, dans le cadre du projet TARMED 2010, à développer avec la FMH une convention sur la gestion des coûts modifiée.

... approuvé le concept sur la recherche concomitante SwissDRG.

... approuvé les mises à jour suggérées par l'Assemblée des délégués concernant le papier de position de la FMH sur SwissDRG.

... approuvé les prises de position suivantes sur:

- l'organe de coordination eHealth;

- l'initiative parlementaire exigeant l'interdiction des mutilations sexuelles;

- l'ordonnance concernant la protection contre la fumée passive;

- l'actualisation de l'ordonnance sur la clause du besoin, avec prise en compte des propositions de l'ASMAC, de la SSMG et de la fmCh;

- la création d'un centre de récolte de données de l'assurance obligatoire des soins pour les assurés dont les prestations ont été suspendues.

... pris position sur la $6^{\mathrm{e}}$ révision de l'AI; le CC refuse la stigmatisation systématique, prévue dans le projet de loi actuel, des personnes souffrant de troubles somatoformes et met en garde contre les attentes démesurées quant au potentiel d'économies de la révision.

... donné, par une lettre d'intention, son accord sur le prix unitaire négocié avec l'OFSP et la CDS, pour la vaccination contre la grippe H1N1. 